\title{
Discovery by Genetic Programming of a Cellular Automata Rule that is Better than any Known Rule for the Majority Classification Problem
}

\author{
David Andre \\ Visiting Scholar \\ Computer Science Dept. \\ Stanford University \\ 860 Live Oak Ave, \#4 \\ Menlo Park, CA 94025 USA \\ andre@flamingo.stanford.edu
}

\author{
Forrest H Bennett III \\ Visiting Scholar \\ Computer Science Dept. \\ Stanford University \\ Stanford, California 94305 \\ fhb3@slip.net
}

\author{
John R. Koza \\ Computer Science Dept. \\ 258 Gates Building \\ Stanford University \\ Stanford, California 94305 \\ koza@cs.stanford.edu
}

\begin{abstract}
It is difficult to program cellular automata. This is especially true when the desired computation requires global communication and global integration of information across great distances in the cellular space. Various human-written algorithms have appeared in the past two decades for the vexatious majority classification task for one-dimensional two-state cellular automata. This paper describes how genetic programming with automatically defined functions evolved a rule for this task with an accuracy of 82.326\%. This level of accuracy exceeds that of the original 1978 GacsKurdyumov-Levin (GKL) rule, all other known human-written rules, and all other known rules produced by automated methods. The rule evolved by genetic programming is qualitatively different from all previous rules in that it employs a larger and more intricate repertoire of domains and particles to represent and communicate information across the cellular space.
\end{abstract}

\section{Introduction}

Massively parallel computation is potentially attractive for solving problems. However, it has proved difficult to program massively parallel computing devices.

This paper describes our research on using genetic programming to evolve programs for cellular automata, which are one type of massively parallel computing system. Specifically, this paper focuses on the well-studied majority classification task for one-dimensional two-state cellular automata. For this problem, genetic programming evolved a rule whose performance exceeds that of the original GacsKurdyumov-Levin (GKL) rule, all other known subsequent human-written rules, and all known rules produced by automated approaches for this problem (table 1).

The result in this paper is that genetic programming has produced a result that is slightly better than human performance for this particular problem. This result joins several similar results recently obtained by both our group and other researchers in applying genetic programming to non-trivial problem domains. The transmembrane segment identification problem is another problem where genetic programming is competitive with human performance. The goal in that problem is to classify a given protein segment (i.e., a subsequence of amino acid residues from a protein sequence) as being a transmembrane domain or nontransmembrane area of the protein. The goal is to perform the classification without using the biochemical foreknowledge concerning hydrophobicity that is typically used in human-written algorithms for this problem. Four different versions of genetic programming have been applied to this problem (Koza 1994a; Koza and Andre 1996a, 1996b). The performance of all four versions using genetic programming is slightly superior to that of algorithms written by knowledgeable human investigators.

A second example involves the problem of automatically discovering patterns in databases of DNA and protein sequences. Genetic programming successfully evolved motifs for detecting the D-E-A-D box family of proteins and for detecting the manganese superoxide dismutase family. Both motifs were evolved without prespecifying their length. Both relied on automatically defined functions to capture common subexpressions.

Table 1 Out-of-sample performance of various rules for the majority classification task.

\begin{tabular}{|l|l|l|}
\hline Rule & Accuracy & Test cases \\
\hline $\begin{array}{l}\text { Best rule evolved by bit-string } \\
\text { genetic algorithm (Das, } \\
\text { Mitchell, and Crutchfield 1994) }\end{array}$ & $76.9 \%$ & $10^{6}$ \\
\hline GKL 1978 human-written & $81.6 \%$ & $10^{6}$ \\
\hline Davis 1995 human-written & $81.8 \%$ & $10^{6}$ \\
\hline Das 1995 human-written & $82.178 \%$ & $10^{7}$ \\
\hline
\end{tabular}




\begin{tabular}{|l|l|l|}
\hline $\begin{array}{l}\text { Best rule evolved by genetic } \\
\text { programming (in this paper) }\end{array}$ & $82.326 \%$ & $10^{7}$ \\
\hline
\end{tabular}

The two genetically evolved consensus motifs detect the two families either as well as, or slightly better than, the comparable human-written motifs found in the PROSITE database (Koza and Andre 1996c).

A third instance involves the automated discovery of an analog electrical circuit for a difficult-to-design asymmetric bandpass filter (Koza, Bennett, Andre, and Keane 1996 in this volume). Other instances have been reported in Kinnear 1994 and Angeline and Kinnear 1996.

Section 2 of this paper provides background on cellular automata. Section 3 presents the majority classification task and previous work on it. Section 4 details the steps required to use genetic programming on the majority classification problem. Section 5 examines the results of our most successful run using genetic programming. In section 6, we examine, in detail, the intricate communication patterns employed by the best rule evolved by genetic programming for this problem.

\section{Cellular Automata}

Local rules govern the important interactions of many animate and inanimate entities. It has been frequently observed that the simultaneous execution of a single relatively simple rule at many local sites leads to the emergence of interesting and complex global behavior (Langton 1989). This global behavior often occurs when entities that have access only to information about their immediate local environment engage in long-distance communication and long-distance integration of information.

Cellular automata are an abstract way of studying and analyzing the simultaneous execution of local rules (Burks 1970; Farmer, Toffoli, and Wolfram 1983; Wolfram 1986; Gutowitz 1991; Wuensche and Lesser 1992).

A cellular space is a uniform array of cells arranged in a certain topological arrangement in a certain number of dimensions. In a cellular automaton (CA), each cell in a cellular space is occupied by an identical automaton (each with its own current state). The next state of each individual automaton in the cellular space depends on its own current state and on the current states of the other automata in a specified local neighborhood around the individual automaton. The state of each automaton at time 0 is called its initial condition.

The best-known cellular automata system is John Conway's game of Life (Berlekamp, Conway, and Guy 1985). It involves a two-dimensional arrangement of identical two-state automata. In the game of Life, the next state of each automaton depends on its current state and on the states of its eight immediately adjacent neighbors in the two-dimensional cellular space.

For a one-dimensional cellular automata, the cellular space is a linear arrangement of identical automata. The next state of each individual automaton might depend on the current state of that automaton and the current states of its neighbors at a particular specified distance. For example, if the distance is three, we denote the seven states that determine the next state of a given automaton as X (for the automaton at the center), $\mathrm{W}$ (the adjacent automaton to the west), E (the adjacent automaton to the east), WW (the automaton at distance 2 to the west), EE, WWW, and EEE. A cellular space is said to have periodic boundary conditions when the cellular space is toroidal. If the automaton located in each cell has only two states, the state-transition function of the automaton is a Boolean function of its own current state and the states of its neighbors at the specified distance.

Cellular automata are dynamical systems that are discrete in time, in space (the cells), and in site value (the states). Cellular automata are the discrete counterparts of continuous dynamical systems defined by partial differential equations and the physicist's concept of field (Toffoli and Margolus 1987). Simulations of fluid flow and other complex processes that depend only on local states are well suited to cellular automata.

It is extremely difficult, in general, to design a single state-transition rule that, when it operates in each cell of the cellular space, produces a desired global behavior.

Genetic algorithms (Holland 1975) operating on fixedlength character strings have been previously used to evolve the initial conditions and state-transition rules for cellular automata (Meyer, Richards, and Packard 1991). Genetic programming (Koza 1992, 1994a, 1994b; Koza and Rice 1992) has been previously used for automatic programming of cellular automata (Koza 1992).

\section{The Majority Classification Problem}

The majority classification problem is one vehicle for exploring how complex calculations can be performed over large expanses of time and space using rules of interaction that operate over a relatively small distance.

In one frequently studied version of this vexatious problem, there is a one-dimensional linear arrangement of 149 two-state automata whose update rule operates on information within a distance of three. The initial states of all 149 automata (called the initial configuration) are the inputs to the calculation. If all 149 automata relax to a common state ( 0 or 1$)$ after a certain amount of time, the common state is considered to be the binary output of the calculation. For the majority classification task, a "0" is correct if a majority of the 149 initial bits are 0 and a "1" is correct if a majority of the bits are 1 . Thus, to solve this problem, a seven-argument Boolean transition rule must be found such that when it is situated at all 149 cells of a onedimensional cellular automata system, the automata converge to the correct configuration of 1490 's or 1491 's (after, say, 600 time steps). The fitness of a rule for this task is measured by its ability to correctly classify any arbitrary random initial state.

It is difficult to construct a two-state, sevenneighborhood cellular automata rule that performs this majority classification task on a 149-bit input configuration. Seven bits cannot store an integer as large as 149 (if, for 
example, one were storing and transmitting a signal communicating a locally observed surplus or deficit of 0 's or 1's ). It is unknown whether a perfect solution to this problem exists.

The difficulty that human programmers have had with this task is indicated by its history. In 1978, Gacs, Kurdyumov, and Levin developed a two-state, sevenneighbor rule for the purpose of studying reliable computation under random perturbations. This GacsKurdyumov-Levin (GKL) rule performs the majority classification task reasonably well. The GKL rule is successful on $81.6 \%$ of the inputs consisting of a configuration of 149 bits (each chosen independently with $50 \%$ probability). Gonzaga de Sa and Maes (1992) showed that this system does indeed relax to a common state.

The majority classification task has been the subject of extensive study (Mitchell, Hraber, and Crutchfield 1993; Das, Mitchell, and Crutchfield 1994; Mitchell, Crutchfield, and Hraber 1994; Crutchfield and Mitchell 1995; Das, Crutchfield, Mitchell, and Hanson 1995; Mitchell 1996).

In 1993, Lawrence Davis (1995) cleverly modified the GKL rule and created a rule that achieved an accuracy of about 81.8\%. Rajarshi Das(1995) created another clever rule that achieved slightly better accuracy than the GKL rule and the Davis rule. The Das rule has an accuracy of about $82.178 \%$. Table 2 shows the GKL rule, the Davis rule, the Das rule, and the rule evolved by genetic programming in this paper. The 128 bits are presented in the natural order that they would appear in a state transition table starting with state 0000000 and ending at state 1111111.

Das, Mitchell, and Crutchfield (1994) evolved rules using a version of the genetic algorithm operating on fixedlength strings. These rules sometimes exhibited qualitatively the same behavior as the GKL rule. However, none of the rules evolved using the genetic algorithm operating on fixed-length strings were as accurate as the original GKL rule. As Das, Mitchell, and Crutchfield (1994) reported, the genetic algorithm usually found only relatively uninteresting block-expanding rules that score in the range of $65-70 \%$ accuracy. The best result from the genetic algorithm operating on fixed-length strings had $76.9 \%$ accuracy. This reported performance may have been the consequence of factors such as the small population size employed.

Land and Belew (1995) suggested that evolution may actually be hindered by the standard representation of the cellular automata rule as a chromosome string of length 128 used in previous work using the genetic algorithm. They suggest that higher-level representations (such as conditionaction pairs) may aid the evolutionary process because of the higher degree of locality in the condition-action pairs. Given Land and Belew's (1995) view, the tree representation employed by genetic programming seems well suited for this task. The size and shape of the ultimate solution is free to undergo evolution in genetic programming. The search strategy employed by genetic programming (and the genetic algorithm) is also important for this problem.

Although many Boolean problems can be trivially solved by hillclimbing methods in a variety of representations, such methods work only when the fitness cases (i.e., the $2^{7}$ lines in the truth table) are independent. When a Boolean function is used for a cellular automata rule, there is no correct answer for a given set of inputs that is independent from the answers for the other inputs. Also, genetic programming supports automatically defined functions, whereas the conventional genetic algorithm operating on fixed-length strings does not have a similar facility for exploiting regularities, symmetries, homogeneities, and modularities of the problem environment.

\section{Preparatory Steps}

The runs reported here used standard genetic programming with automatically defined functions (Koza 1994a) as summarized in table 3. The problem (coded in ANSI C) was run on a medium-grained parallel Parsytec computer system consisting of $6480-\mathrm{MHz}$ Power PC 601 processors arranged in a toroidal mesh with a host PC Pentium type computer. The so-called distributed genetic algorithm or island model for parallelization was used. That is, subpopulations (demes) were situated at the processing nodes of the system. Population size was $Q=800$ at each of the $D=64$ demes for a total population size of 51,200 . The initial random subpopulations were created locally at each processing node. Generations were run asynchronously on each node. After a generation of genetic operations was performed locally on each node, four boatloads, each consisting of $B=3 \%$ (the migration rate) of the subpopulation (selected on the basis of fitness) were dispatched to each of the four toroidally adjacent nodes. Details of the parallel implementation of genetic programming can be found in Andre and Koza 1996. 
Table 2 The succession of "best" cellular automata rules for the majority classification task, presented in truth table order from 0000000 to 1111111 (i.e. 0 to 127).

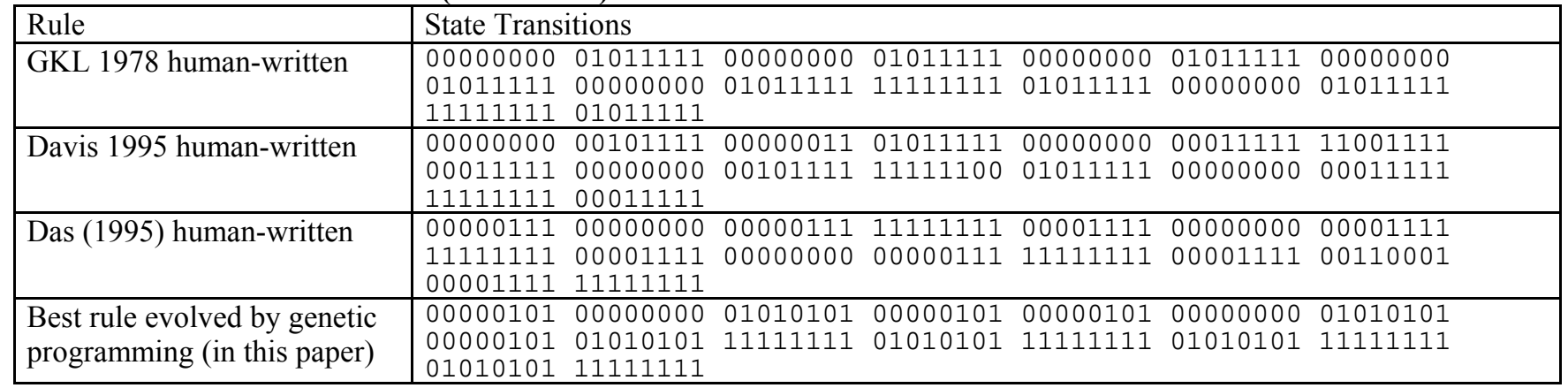


Table 3 Tableau for the majority classification problem for one-dimensional cellular automata.

\begin{tabular}{|c|c|}
\hline Objective: & $\begin{array}{l}\text { Find a seven argument Boolean function that performs the majority classification problem } \\
\text { for a } 149 \text {-width one-dimensional cellular automata system. }\end{array}$ \\
\hline $\begin{array}{l}\text { Architecture of the overall } \\
\text { program with ADFs: }\end{array}$ & $\begin{array}{l}\text { One result-producing branch and one two-argument automatically defined function, } \mathrm{ADF} 0 \text {, } \\
\text { and one three-argument automatically defined function, } \mathrm{ADF} 1 \text {. ADF } 1 \text { can refer to } \mathrm{ADF} 0 \text {. }\end{array}$ \\
\hline Terminal set for the RPB: & $\mathrm{X}, \mathrm{E}, \mathrm{EE}, \mathrm{EEE}, \mathrm{W}, \mathrm{WW}$, and WWW. \\
\hline Function set for the RPB: & AND, OR, NAND, NOR, NOT, IF, XOR, ADF 0, and ADF 1. \\
\hline Terminal set for ADF 0: & $\mathrm{X}, \mathrm{E}, \mathrm{EE}, \mathrm{EEE}, \mathrm{W}, \mathrm{WW}, \mathrm{WWW}, \mathrm{ARG} 0$, and ARG1. \\
\hline Function set for ADF 0: & AND, OR, NAND, NOR, NOT, IF, and XOR. \\
\hline Terminal set for ADF 1: & $\mathrm{X}, \mathrm{E}, \mathrm{EE}, \mathrm{EEE}, \mathrm{W}, \mathrm{WW}, \mathrm{WWW}, \mathrm{ARG} 0, \mathrm{ARG} 1$, and ARG2. \\
\hline Function set for ADF 1: & AND, OR, NAND, NOR, NOT, IF, XOR, and ADF 0. \\
\hline Fitness cases: & $\begin{array}{l}\text { - } 1,000149 \text {-bit initial configurations were used as in-sample fitness cases. These initial } \\
\text { configurations were created randomly, with no bias (i.e., } 0 \text { and } 1 \text { each have an independent } \\
50 \% \text { probability of being chosen). Thus, the distribution of densities of the initial state } \\
\text { vectors was a binomial distribution centered at } 0.50 \text {. } \\
\text { - The out-of-sample fitness cases consisted of } 1,000,000 \text { (and later } 10,000,000 \text { and } \\
15,000,000 \text { ) similarly created initial configurations. }\end{array}$ \\
\hline Raw fitness: & $\begin{array}{l}1,000 \text { minus the number of fitness cases for which the system relaxes to the correct } \\
\text { configuration after } 600 \text { time steps. }\end{array}$ \\
\hline Standardized fitness: & 1,000 minus raw fitness. \\
\hline Hits: & Raw fitness. \\
\hline Parameters: & $\begin{array}{l}\text { - Population size, } M \text {, is } 51,200 \text { ( } 64 \text { times } 800) \text {. } \\
\text { - Maximum number of generations to be run, } G \text {, is } 51 \text {. } \\
\text { - } 89 \% \text { crossovers, } 10 \% \text { reproductions, and } 1 \% \text { mutations were used on each generation. } \\
\text { - A maximum of } 500 \text { points (functions and terminals) for the result-producing branch and } \\
250 \text { points for each automatically defined function was allowed. } \\
\text { - Structure-preserving crossover with branch typing was used. } \\
\text { - The other parameters for controlling the runs of genetic programming were the default } \\
\text { values specified in Koza }(1994 \mathrm{a}) \text {. }\end{array}$ \\
\hline
\end{tabular}

\section{Emergent Computation in the Most Successful Run}

We made five runs of genetic programming on this problem. Each run produced numerous individuals that were reminiscent of the GKL rule. Each of the runs produced numerous individuals that scored well above the best accuracy of $76.9 \%$ produced by the genetic algorithm operating on fixed-length character strings. The best rule evolved by genetic programming scores better than any known human-written or machine-produced rule (table 1).

An examination of the most successful run illustrates the emergence of many interesting entities prior to the creation of the best individual.

The best individual program from the initial random generation indiscriminately classifies all fitness cases as having a majority of 1 's. Since 525 of the 1,000 randomly created fitness cases on this particular run happened to have a majority of 1's, this best of generation 0 scores 525 hits.

The best individual of generation 1 scores 650 hits. The activity of a one-dimensional cellular automata system can be presented as a two dimensional grid in which the top horizontal row contains the states ( 0 or 1$)$ of the 149 automata at a time 0 (i.e., the initial configuration of the system) and in which each successive row represents the states of the 149 automata at successive time steps. Figure 1 shows a small part of such a diagram for this individual. In the space-time diagram, we see large areas dominated by solid blocks of repeated simple regular patterns, called domains, where a given domain is specified by a regular expression. The two domains shown in figure 1 are the domain denoted by the regular expression $1^{*}$ and the domain denoted by the expression $0^{*}$. The space-time diagrams in this paper display 1's as black, and 0's as white. Thus we call the $1^{*}$ domain black (designated by (B)) and the $0 *$ domain white (designated by $(\mathbf{W})$ ).

The domains of this generation 1 individual interact in various ways. When a domain consisting of a solid block of 1 's is to the left of a domain consisting of a solid block of 0 's, the interface consists of ...111000... and the corresponding positions at the next time step also consist of ...111000... . Because these particular two domains do not move left or right over time, this particular domain interface is said to have a velocity of zero.

Following Das, Mitchell, and Crutchfield (1994), we describe such an interface between two domains as a particle, denoted $\mathrm{P}(\mathrm{xy})$, where $\mathrm{x}$ is the domain on the left and $y$ the domain on the right. Particles are one of the ways that information is communicated across large distances in time and space in a cellular space. In figure 1, line a shows 
the path of the $\mathrm{P}(\mathrm{BW})$ particle with a velocity of 0 and line b shows the path of the $\mathrm{P}(\mathrm{WB})$ particle with a velocity of $1 / 2$.

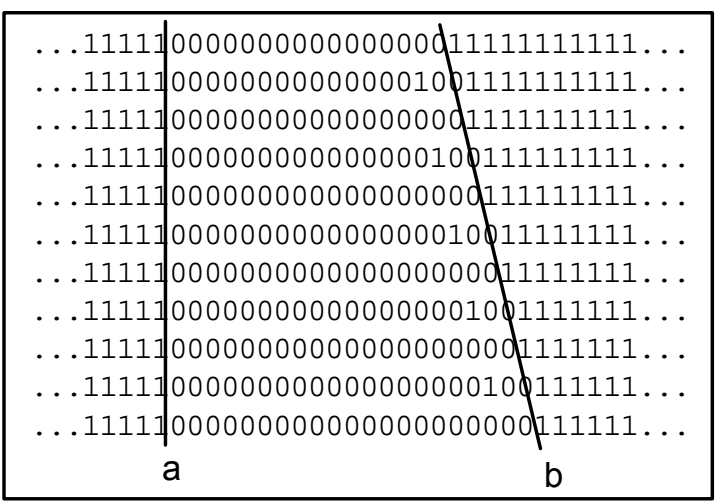

Figure 1 Eleven time steps of the partial space-time behavior of the best individual of generation 1 on one fitness case showing the spread of the zero domain.

This evolved rule from generation 1 overpredicts a majority of 1 's and underpredicts a majority of 0 's. This individual is an example of what Mitchell, Hraber, and Crutchfield (1993) called a block-expanding rule (i.e. a rule that converges to a state unless a sufficiently large block of adjacent or nearly adjacent instances of the opposite state exists in the input).

The best program of generation 6 scores 706 hits on the 1,000 in-sample fitness cases. A portion of its space-time behavior is shown in figure 2 . The behavior of this individual is similar to the individual of generation 1 . However, it scores slightly better because it has modified the conditions under which it expands a block. The interaction between the domain of zeros on the left and ones on the right is quite complex in this individual, and the interface between the two domains is quite large. The particle $\mathrm{P}(\mathrm{WB})$ travels at a velocity of $2 / 7$ in this individual.

The best individual of generation 15 scores 815 hits. Its behavior (see figure 3) is somewhat similar to that of the GKL rule, although it scores less than $80 \%$ on out-ofsample fitness cases. Like the GKL rule (figure 7), this rule separates white on the left from black on the right with a

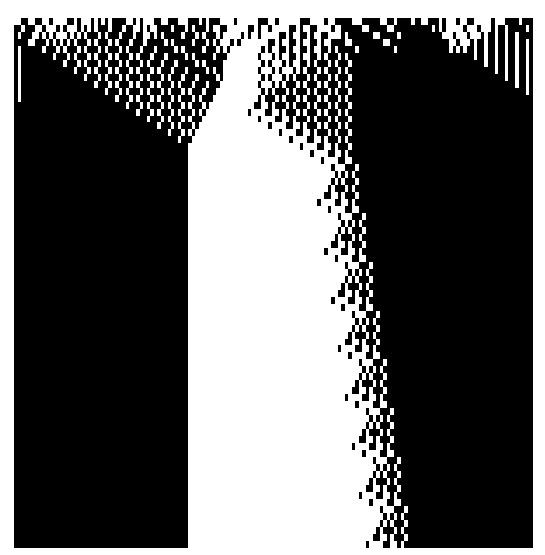

Figure 2 Partial space-time behavior of the best individual of generation 6. Black represents $1 \mathrm{~s}$, white $0 \mathrm{~s}$. gray domain that grows into both black and white. When black is to the left of white, a zero-velocity particle characterizes their interaction. The combination of these two

concepts yields the basic mechanism that both GKL and this generation 15 individual use to compute global measures of density. If the domain of all white is larger, it will win out over black, and vice versa.

Although similar to GKL, the rule of generation 15 has several new gray domains that are not quite the same as the gray domain in the GKL rule's behavior. The new domains interact, as can be seen in figure 4. In this example, the zero and one domains do not enter into the computation until the very end - all of the primary computation is performed by the new gray domains. At this point, however, the new gray domains do not interact particularly well - the rule fails often when it computes with the new gray domains.

The best individual of the run emerged on generation 17. The 36-point result-producing branch is

(nand (xor (or (adfo x (not WWW)) (not WWW)) (adfO (adfO E X) (or E W)) ) (or (nand (if EEE $\mathrm{E} X)$ (if $\mathrm{EEE} W$ (and $\mathrm{EE} W W W)$ )) (adfl (nand $W$ E) (xor WWW W) E))).

The 10-point automatically defined function $\mathrm{ADF} O$ is (if (nor (or X X) (nor arg1 $\arg 0)$ ) EEE WWW).

The 44-point automatically defined function $\mathrm{ADF} 1$ is

(xor $W$ (xor (adfo (adfo (if (or (not $W$ ) (adfo WWW $\arg 2)$ ) (xor (adf0 WWW X) (nor WW WWW)) (nand (xor X X) (or X WW))) (nor (or (if arg2 EEE $\arg 2)(\operatorname{nor} \arg 2 \arg 1))(\operatorname{not}(\operatorname{xor} W E))))$ (xor W (not $\arg 2)))$ EEE)) .

This rule scores 824 hits on the 1,000 in-sample fitness cases, and scores $82.4 \%$ accuracy over 100,000 out-ofsample cases. It scores $82.326 \%$ accuracy over $10,000,000$ additional cases.

This accuracy is slightly better than the score of the GKL rule. We are aware of two human-coded rules that also are better than the GKL rule - the Das rule (1995) and the Davis rule (1995) (table 2). We tested the best individual evolved by genetic programming against each of the other three rules using the same fitness cases $\left(10^{6} 10^{7}\right.$ fitness cases). Table 1 shows the results. As can be seen, the best rule evolved by genetic programming is slightly better than

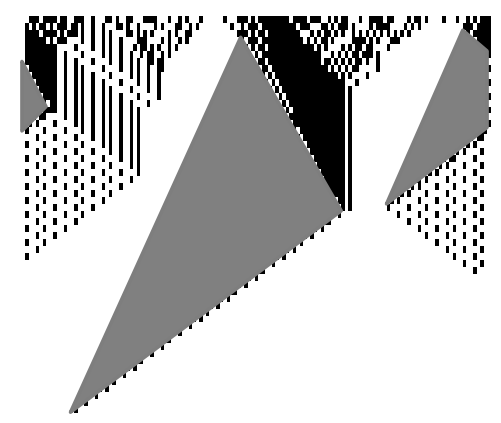

Figure 3 Space-time behavior of the best individual of generation 15 on a typical initial state vector. 


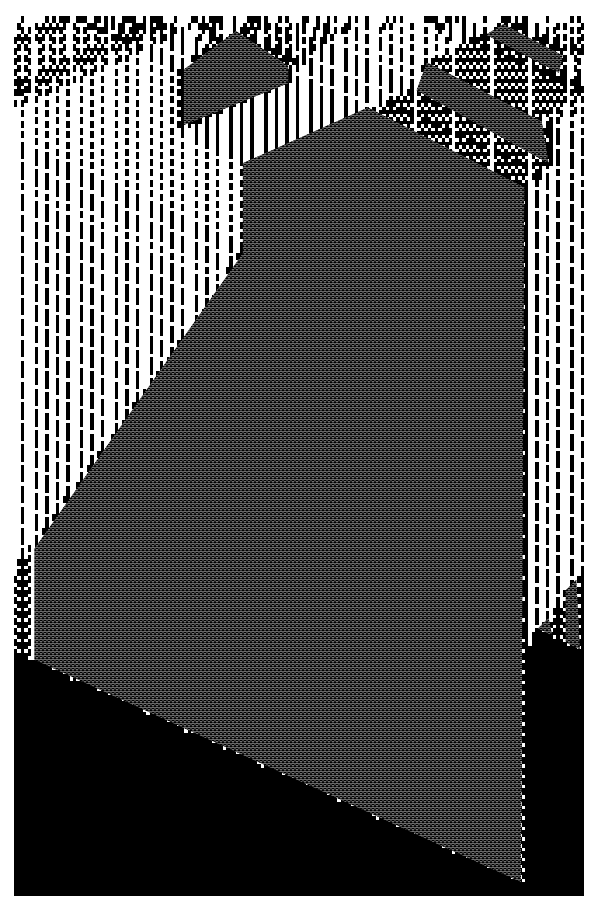

Figure 4 Space-time behavior of the best individual of generation 15 where there are no all black or all white domains until the end.

all the other rules. Non-parametric $\chi 2$ tests with one degree of freedom were performed; the $\operatorname{probability}(p)$ that the pairwise differences between the best rule evolved by genetic programming and each of the other three rules were attributable to chance was less than 0.001 (i.e. the differences are statistically significant, $p<0.001$ ).

\section{The Best Rule Evolved by Genetic Programming}

Why does the best rule evolved by genetic programming score better than the previously known (human-written) rules? First, it has more domains than the GKL rule. The 11 domains of the rule evolved by genetic programming (table 4) classify the density of 1 's into finer levels of gray than do the black, white, and checkerboard gray domains of the GKL rule (analyzed in detail in Das, Mitchell, and Crutchfield 1994). The checkerboard gray domain of the GKL rule corresponds to the regular expression $(10)^{\star} \approx$ (01) * (Das, Mitchell, and Crutchfield 1994). In addition, the rule evolved by genetic programming employs a larger number of particles. The rule evolved by genetic programming employs 10 particles that are identical to the particles (Das, Mitchell, and Crutchfield 1994) in the GKL rule; however, it also discovered at least 40 additional particles that involve the new gray domains. All the new particles have velocity $0,+3$, or -3 .

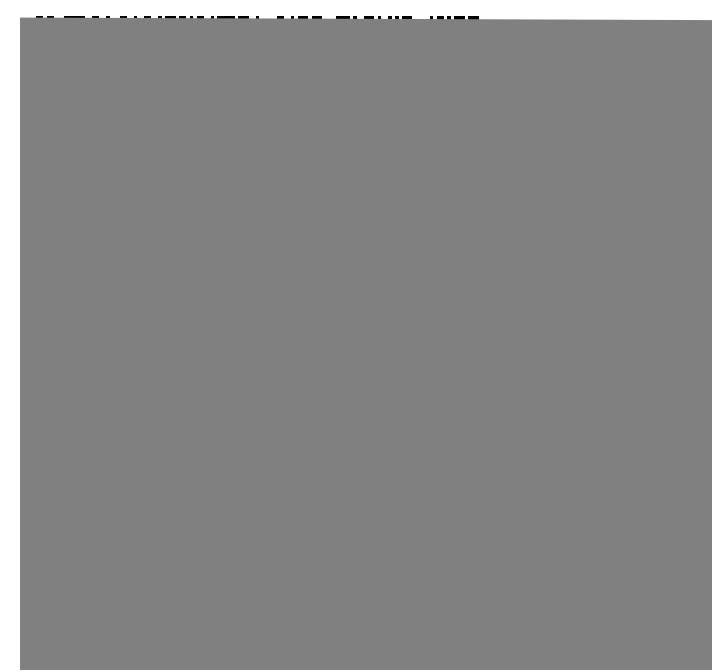

Figure 5 Space-time diagram showing the behavior of the best rule evolved by genetic programming. The differences between the shades of gray and the area highlighted by the arrow are important to convergence.

More domains would be purely a superficial difference if the new domains did not participate in the computation. However, the new gray domains are crucial to the computations being performed by the genetically evolved rule. In the behavior shown in figure 5, for example, the new grays are critical to the success of the rule. If all the grays acted identically, then the automata would not converge in this example. The interactions of the new gray domains in the area indicated by the arrow allow the white domain to escape and encroach to the left.

In addition, there are periods (as shown in figure 6) during the behavior of the best rule evolved by genetic programming where none of the GKL domains exist. In fact, there are periods in which the computation is carried out entirely by the new gray domains.

Even though the new gray domains take part in the computation, one might question whether they account for

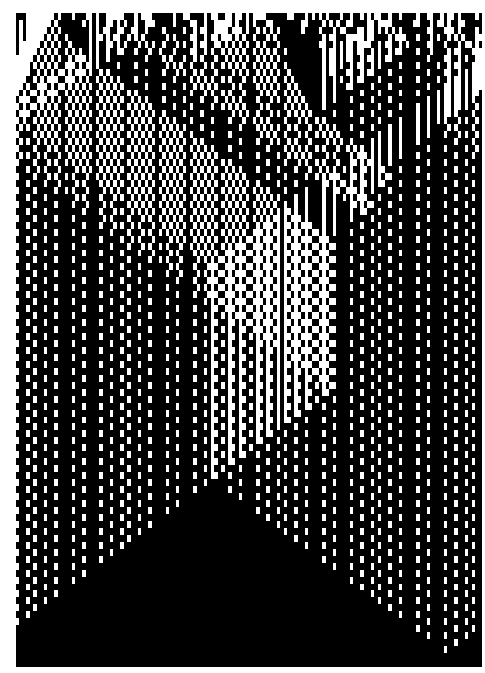

Figure 6 The behavior of the best rule evolved by genetic programming on one set of initial states. 
the difference in score between the best rule evolved by genetic programming and the GKL rule.

However, the behavior of both rules shown in figure 7 indicates that the finer levels of gray do confer an advantage on the best rule evolved by genetic programming. On the left of the initial state vector, there are two fairly large regions of white $(0 \mathrm{~s})$ separated by a region of black (1s) marked in the diagrams by the arrows. Under the GKL rule, the black area is eliminated by the two adjacent white domains. In the best rule evolved by genetic programming, these two white areas are classified as light gray domains and the black area becomes a dark gray domain (all shifted

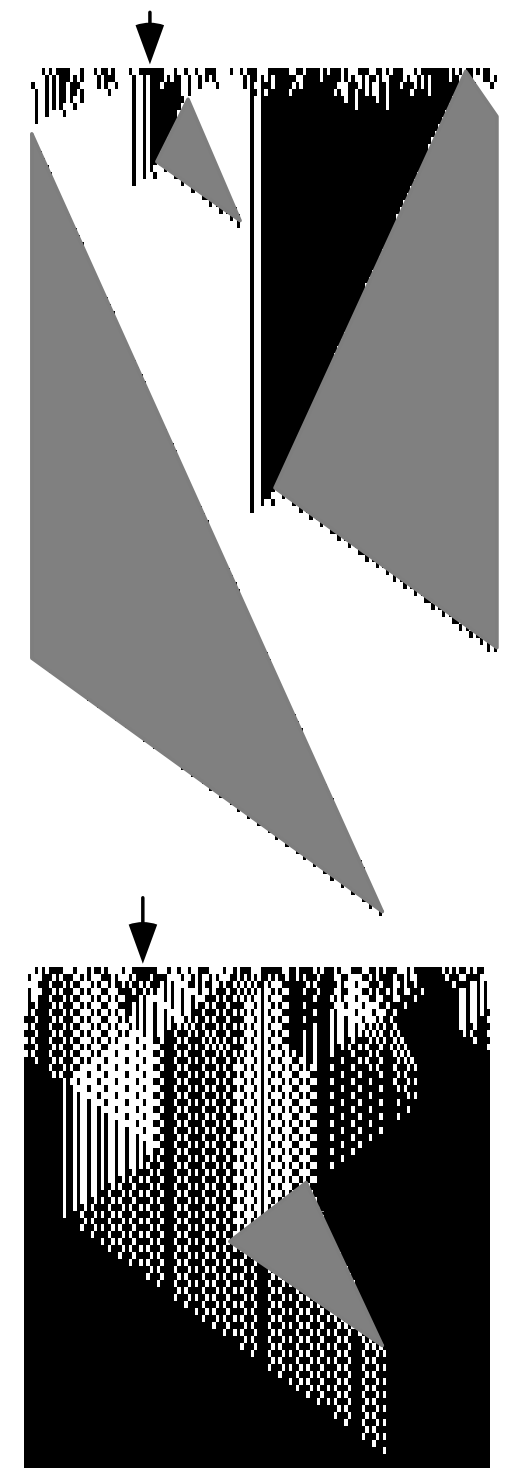

Figure 7 The space-time behavior of the best rule evolved by genetic programming (bottom) and the GKL rule (top) on the same initial state vector. The ability of the evolved rule to classify domains of the input as intermediate gray values allows it to avoid the 'round-off' error that the GKL makes. to the right slightly). Because it can make use of extra domains, the best rule evolved by genetic programming retains the information that there is a significant density of black (1s) in the vicinity of the arrow until additional global information can resolve the local dispute.

The basic mechanism of best rule evolved by genetic programming is similar to that of the GKL rule in that there is a race among the black, white, and gray domains. Figure 8 shows a space-time diagram of the behavior of the best rule evolved by genetic programming.

In the best rule evolved by genetic programming, a white domain $(\mathbf{W})$ is separated on the right from a black domain (B) by a growing domain of checkerboard gray (6). The white domain $(\mathbf{W})$ is separated on the left from the black domain (B) by one or both of the separator domains, (3) and (8), which are domains whose interactions with either white or black have zero velocity. In the case shown in figure 8, the (B) domain is larger, and the gray (6) domain cuts off the white domain $(\mathbf{W})$, allowing the black domain (B) to break through. In this case, the extra gray domains of (7) and (9) are not important, but they play a role in other fitness cases.

The mechanism discussed above is sufficient for many cases, but fails to handle the case when the black and white domains are of approximately equal size. In this circumstance, the gray (6) domain cuts off both the white and black domains. In the behavior of the GKL rule, when there is such a 'tie', after a brief transition, the black and white domains seem to 'swap' places, separated by a new growing gray domain (figure 9).

In the behavior of the best rule evolved by genetic programming such collisions occur slightly differently and yield different behavior after the collision. Under this rule, the black (B) and white $(\mathbf{W})$ domains are separated by a potentially larger distance by one or both of the separator

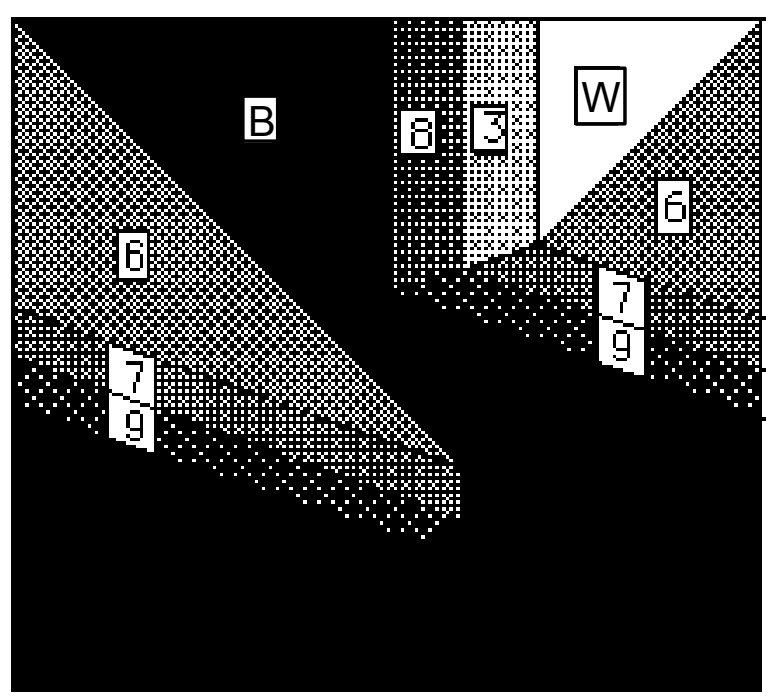

Figure 8 Abstracted Space-Time domain diagram for the best rule evolved by genetic programming showing its typical behavior. The darkness of each domain corresponds to the density of ones. 


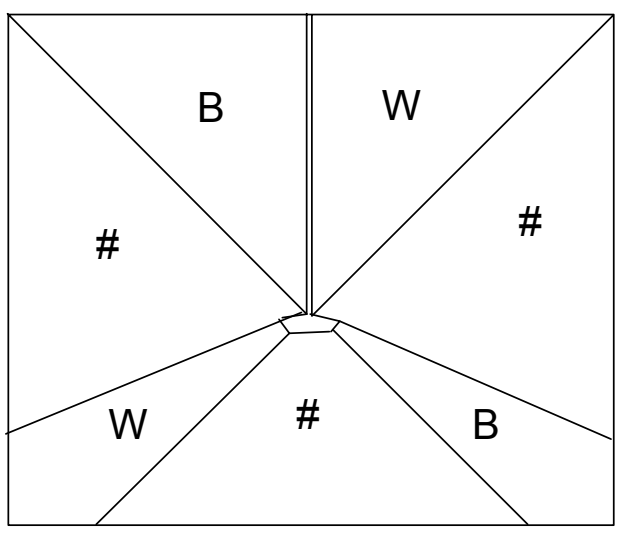

Figure 9 Collisions under GKL rule.

domains (8) and (3). If there is a tie, new domains emerge after the collision instead of the black and white domains, and there is no immediate gray separator domain. The new gray domains of (7) and (9) appear to represent the continuation of the black (B) domain, whereas the (2) and (1) domains appear to represent the continuation of the white (W) domain. Thus, when there is a tie in the best rule evolved by genetic programming; the original domains of black and white disappear completely; and the computation is carried out entirely by the gray domains. The abstract schematic for this interaction is shown in figure 10 .

One problem that a rule utilizing zero-velocity particles can have is that the initial state vector could either be in a state or relax to a state where convergence does not take place. If the automata either starts in or reaches a state where the automata is filled with domains that have zerovelocity interactions with the adjacent patterns, the automata cannot converge. Thus, one potential downside to the best rule evolved by genetic programming is that because it utilizes more domains in its computation that have zero-velocity particles, it may be more likely to be trapped in a non-convergent steady-state. We found only one such input pattern in our testing (see figure 11). Given that the best rule evolved by genetic programming scores better than any other known rule, however, it is possible

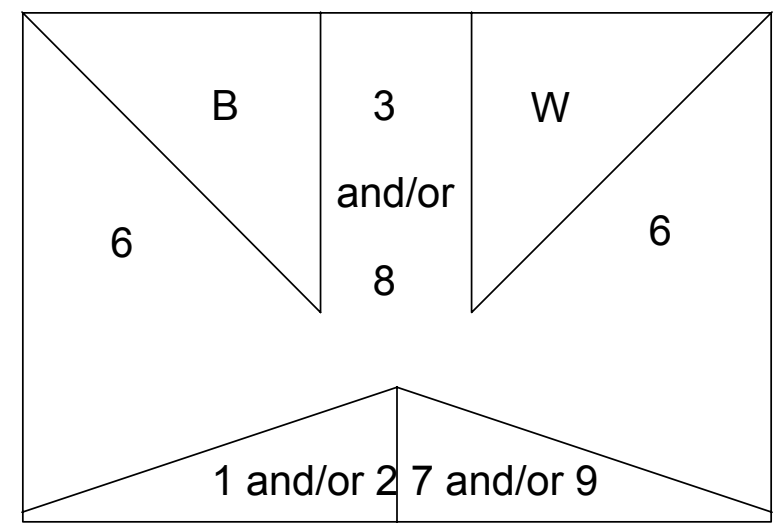

Figure 10 Collisions under the best rule evolved by genetic programming.

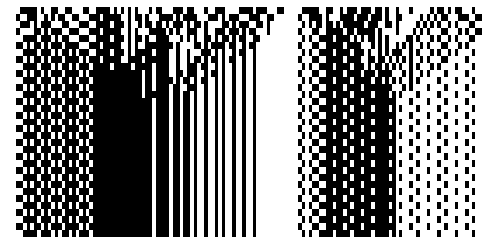

Figure 11 The behavior of the best rule evolved by genetic programming on an initial state vector where the automata does not converge.

that the occasional non-convergence represents a strategic tradeoff rather than a defect.

The asymmetry of the best rule evolved by genetic programming suggests that there may be other rules that are better yet at performing the majority classification task.

\section{Conclusions}

A cellular automata rule for the majority classification task was evolved using genetic programming with automatically defined functions. This genetically evolved rule has an accuracy of $82.326 \%$. This accuracy exceeds that of the Gacs-Kurdyumov-Levin (GKL) rule, all other known human-written rules, and all other known rules produced by previous automated approaches. The best rule evolved by genetic programming differs qualitatively from these other rules. It utilizes a very fine internal representation of density information; it employs a large number of different domains and particles; and it uses an intricate set of signals for communicating information over large distances in time and space.

\section{Acknowledgements}

The authors gratefully thank Lawrence Davis, Melanie Mitchell, and James Crutchfield for helpful discussions and for reviewing a draft of this paper. Simon Handley and Scott Brave made helpful comments on drafts of this paper.

\section{Bibliography}

Andre, David and Koza, John R. 1996. Parallel genetic programming: A scalable implementation using the transputer architecture. In Angeline, Peter J. and Kinnear, Kenneth E. Jr. (editors). Advances in Genetic Programming 2. Cambridge, MA: The MIT Press.

Angeline, Peter J. and Kinnear, Kenneth E. Jr. (editors). Advances in Genetic Programming 2. Cambridge, MA: The MIT Press.

Burks, Arthur W. 1970. Essays on Cellular Automata. Urbana, IL: University of Illinois Press.

Crutchfield, J. P. and Mitchell, Melanie 1995. The evolution of emergent computation. Proceedings of the National Academy of Sciences, USA. 92 (23).

Das, Rajarshi, Mitchell, Melanie, and Crutchfield, J. P. 1994. A genetic algorithm discovers particle-based computation in cellular automata. In Davidor, Yuval, Schwefel, Hans-Paul, and Maenner, Reinhard (editors). 1994. Parallel Problem Solving from Nature - PPSN III. (Lecture Notes in Computer Science, Volume 866). Berlin: Springer-Verlag. 344-353. 
Das, Rajarshi, Crutchfield, J. P., Mitchell, Melanie, and Hanson, J. E. 1995. Evolving globally synchronized cellular automata. In Eshelman, Larry J. (editor). Proceedings of the Sixth International Conference on Genetic Algorithms. San Francisco, CA: Morgan Kaufmann Publishers.

Das, Rajarshi. 1995. Personal communication.

Davis, Lawrence. 1995. Personal communication.

Farmer, Doyne, Toffoli, Tommaso, and Wolfram, Stephen (editors). 1983. Cellular Automata: Proceeding of an Interdisciplinary Workshop, Los Alamos, New Mexico, March 7-11, 1983. Amsterdam: North-Holland Physics Publishing. Also in Physica D, volume 10.

Gacs, P., Kurdyumov, G. L., and Levin, L. A. 1978. One dimensional uniform arrays that wash out finite islands. Problemy Peredachi Informatsii. 12(1978) 92 - 98.

Gardner, Martin 1970. The fantastic combinations of John Conway's new solitaire game "Life." Scientific American. 223(April): 120-123.

Gonzaga de Sa, P. and Maes. C. 1992. The Gacs-KurdyumovLevin automaton revisited. Journal of Statistical Physics. 67(3/4) 507-522.

Gutowitz, Howard (editor). 1991. Cellular Automata: Theory and Experiment. Cambridge, MA: The MIT Press. Also in Physica D 1990.

Holland, John H. 1975. Adaptation in Natural and Artificial System. Ann Arbor, MI: University of Michigan Press. The 1992 second edition was published by The MIT Press.

Kinnear, Kenneth E. Jr. (editor). Advances in Genetic Programming. Cambridge, MA: The MIT Press 1994.

Koza, John R. 1992. Genetic Programming: On the Programming of Computers by Means of Natural Selection. Cambridge, MA: The MIT Press.

Koza, John R. 1994a. Genetic Programming II: Automatic Discovery of Reusable Programs. Cambridge, MA: The MIT Press.

Koza, John R. 1994b. Genetic Programming II Videotape: The Next Generation. Cambridge, MA: The MIT Press.

Koza, John R. and Andre, David. 1996a. Classifying protein segments as transmembrane domains using architecture-altering operations in genetic programming. In Angeline, Peter J. and Kinnear, Kenneth E. Jr. (editors). 1996. Advances in Genetic Programming II. Cambridge, MA: MIT Press. In Press.

Koza, John R. and Andre, David. 1996b. Evolution of iteration in genetic programming. In Evolutionary Programming $V$ : Proceedings of the Fifth Annual Conference on Evolutionary Programming. Cambridge, MA: MIT Press. In Press.
Koza, John R. and Andre, David. 1996c. Automatic discovery of protein motifs using genetic programming. In Yao, Xin (editor). 1996. Evolutionary Computation: Theory and Applications. Singapore: World Scientific. In Press.

Koza, John R., Bennett III, Forrest H, Andre, David, and Keane, Martin A. 1996. Automated WYWIWYG design of both the topology and component values of analog electrical circuits using genetic programming. In Koza, John R., Goldberg, David E., Fogel, David B., and Riolo, Rick L. (editors). Genetic Programming 1996: Proceedings of the First Annual Conference, July 28-31, 1996, Stanford University. Cambridge, MA: MIT Press. In this volume.

Koza, John R., and Rice, James P. 1992. Genetic Programming: The Movie. Cambridge, MA: MIT Press.

Land, Mark and Belew, Richard K. 1995. In McDonnell, John R., Reynolds, Robert G., and Fogel, David B. (editors). Evolutionary Programming IV: Proceedings of the Fourth Annual Conference on Evolutionary Programming. Cambridge, MA: The MIT Press. Pages 403-434.

Langton, Christopher G. (editor). 1989. Artificial Life, Santa Fe Institute Studies in the Sciences of Complexity. Volume VI. Redwood City, CA: Addison-Wesley.

Meyer, Thomas P., Richards, Fred C., and Packard, Norman H. 1989. A learning algorithm for modeling. Physical Review Letters. 63(16) 1735-1738. October 16.

Mitchell, Melanie. 1996. An Introduction to Genetic Algorithms. Cambridge, MA: The MIT Press.

Mitchell, Melanie, Crutchfield, J. P., and Hraber, P. T. 1994. Dynamics, computation, and the "edge of chaos": A reexamination. In G. Cowan, D. Pines, and D. Melzner (editors). Complexity: Metaphors, Models, and Reality. Santa Fe Institute Studies in the Sciences of Complexity, Proceedings, Volume 19. Reading, MA: Addison-Wesley.

Mitchell, Melanie, Hraber, P. T., and Crutchfield, J. P. 1993. Revisiting the edge of chaos: Evolving cellular automata to perform computations. Complex Systems. (7) 89-130.

Toffoli, T. and Margolus, N. 1987. Cellular Automata Machines. Cambridge, MA: The MIT Press.

Wuensche, Andrew and Lesser, Mike. 1992. The Global Dynamics of Cellular Automata. Santa Fe Institute Studies in the Sciences of Complexity. Reference Volume I. Reading, MA: Addison-Wesley.

Wolfram (editor). 1986. Theory and Applications of Cellular Automata. Singapore: World Scientific.

Table 4 The 11 domains of the best rule evolved by genetic programming.

\begin{tabular}{|l|l|l|}
\hline Regular Domain & Domain Name & Color \\
\hline $0^{*}$ & $\mathbf{W}$ & White \\
\hline$(000001)^{*} \approx(100000)^{*} \approx(010000)^{*} \approx(001000)^{*} \approx(000100)^{*} \approx(000010)^{*}$ & $\mathbf{1}$ & Very Light Gray \\
\hline$(000101)^{*} \approx(100010)^{*} \approx(010001)^{*} \approx(101000)^{*} \approx(010100)^{*} \approx(001010)^{*}$ & $\mathbf{2}$ & Light Gray \\
\hline$(001)^{*} \approx(100)^{*} \approx(010)^{*}$ & $\mathbf{3}$ & Light Gray \\
\hline$(001101)^{*} \approx(100110)^{*} \approx(010011)^{*} \approx(101001)^{*} \approx(110100)^{*} \approx(011010)^{*}$ & $\mathbf{4}$ & Gray \\
\hline$(001011)^{*} \approx(100101)^{*} \approx(110010)^{*} \approx(011001)^{*} \approx(101100)^{*} \approx(010110)^{*}$ & $\mathbf{5}$ & Gray \\
\hline$(01)^{*} \approx(10)^{*}$ & $\mathbf{6}$ & Gray \\
\hline$(011101)^{*} \approx(101110)^{*} \approx(010111)^{*} \approx(101011)^{*} \approx(110101)^{*} \approx(111010)^{*}$ & $\mathbf{7}$ & Dark Gray \\
\hline$(011)^{*} \approx(101)^{*} \approx(110)^{*}$ & $\mathbf{8}$ & Dark Gray \\
\hline$(011111)^{*} \approx(101111)^{*} \approx(110111)^{*} \approx(111011)^{*} \approx(111101)^{*} \approx(111110)^{*}$ & $\mathbf{9}$ & Very Dark Gray \\
\hline
\end{tabular}


